\title{
Supporting Healthcare Integration of Refugees Exploiting Reusable Learning Objects: The ASPIRE Framework
}

\author{
Ioanna DRATSIOU ${ }^{\mathrm{a}, 1}$, Annita VARELLA ${ }^{\mathrm{a}}$, Natalia STATHAKAROU ${ }^{\mathrm{b}}$, \\ Stathis KONSTANTINIDIS ${ }^{c}$ and Panagiotis BAMIDIS ${ }^{\mathrm{a}}$ \\ a Laboratory of Medical Physics, School of Medicine, Aristotle University of \\ Thessaloniki, Greece \\ ${ }^{\mathrm{b}}$ Department of Learning Informatics Management and Ethics, \\ Karolinska Institutet, Stockholm, Sweden \\ ${ }^{\mathrm{c}}$ University of Nottingham, UK
}

\begin{abstract}
The significant increase of refugees' migration has demonstrated an overwhelming need for their access and integration to healthcare systems. Elearning, especially in the form of experiential digital learning, has been highlighted as an important ally in supporting refugees' health needs. This paper aims to discuss the design and development process of Reusable Learning Objects (RLOs) to enhance digital health applications for refugees and integrate them to healthcare systems. The participatory approach based on the "ASPIRE" framework was followed. Using the "ASPIRE" process, 9 stakeholders were involved in the first phase of the development process, where a co-creation storyboarding workshop was held in Greece by the Medical Physics Laboratory of the Aristotle University of Thessaloniki. Essential qualitative and quantitative feedback was collected from stakeholders through an online questionnaire administered in Google Forms. Overall, enlightening ideas were expressed and all stakeholders commented positively on the value and efficacy of the workshop.
\end{abstract}

Keywords. Refugees, healthcare, e-learning, Reusable Learning Objects, participatory approach

\section{Introduction}

The mass migration movement of more than 28 million refugees and asylum seekers, reported in 2017, has undoubtedly reached unprecedented levels [1,2]. In the absence of adequate and decent living, refugees and asylum-seekers are arguably confronted with certain challenges to accessing healthcare, including financial, administrative, language and cultural barriers, as well as insufficient understanding of how healthcare is organized and delivered [3]. On the basis of this increased arrival of multicultural low healthliterate refugees, improving their health and reducing health inequalities have been highlighted [4] and in this context, the World Health Organization (WHO) has endorsed digital health crucial in improving health services, particularly for vulnerable populations [5]. Indeed, utilization of digital technology by both refugees and humanitarian aid

\footnotetext{
${ }^{1}$ Corresponding Author, Ioanna Dratsiou; E-mail: idratsiou@gmail.com.
} 
providers has been increasing [6] and United Nations High Commissioner for Refugees (UNHCR) now advocates for the use of technology and e-learning to support refugee health needs [7]. However, digital learning for refugee education is a new field with little research assessing its take-up and impact and there is a knowledge gap of digital health applications for refugees [3]. Certainly, there have been many initiatives for designing and developing e-learning resources and objects, however, they rarely involve stakeholders directly in the design and development process [8].

In this context, ReHIn project [9], a research project on Refugees' Health Integration funded by the Erasmus + Programme of the European Union, aims to raise awareness and support the integration of refugees into the European Union health culture through the creation of web-based educational resources. A set of six Reusable Learning Objects (RLOs) will be developed and integrated into a MOOC (Massive Open Online Course) aiming at the integration in terms of use of the health services, but also in the way that health and medicine is practiced in EU in comparison to major refugees' home countries. The participatory approach "ASPIRE" framework; an acronym that stands for Aims, Storyboarding, Population, Implementation, Release and Evaluation [8] is adopted in the design and development methodology of RLOs exploiting participatory co-design principles and mainly focus on involving all stakeholders on a six-step development process. Figure 1 below illustrates the workflow of the "ASPIRE" framework. RLOs can be considered as standalone learning units, small in size, approximately 5 to 15 minutes long [8] with specific learning objectives that form a comprehensive e-learning experience [10] that can be modified and repurposed [11]. This study aims to discuss the co-creation methodology for the development of a RLO, with respect to the refugees' healthcare integration, using the ASPIRE framework participatory approach.

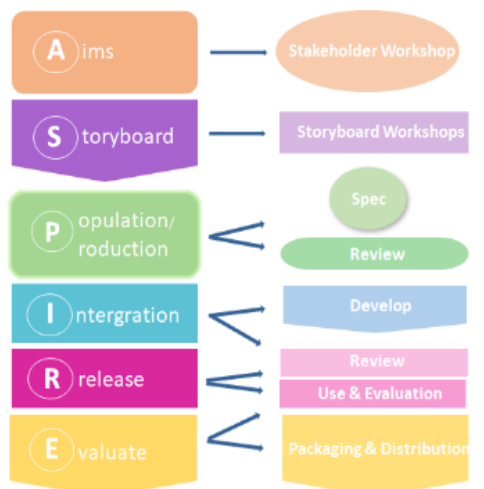

Figure 1. ASPIRE Framework for digital training and education resources [12]

\section{Methods}

\subsection{Needs Analysis}

In the scope of the ReHIn project, a needs analysis was conducted aiming at collecting essential information on the knowledge gaps of the refugees in regard to the healthcare systems and culture. Starting point of this study was the systematic identification and prioritization of the content of the RLOs. In particular, a workshop was conducted in November'19 at Karolinska Institutet, Sweden, where stakeholders' views were 
explored. This study involved 15 stakeholders that represented non-governmental organizations (NGOs), policy makers, governmental organizations and researchers in this field. The input of the participants was gathered through questionnaires and focus groups. The results revealed that the topics considered most important were: access to the healthcare system; knowledge of rights; women's rights and reproductive health; health literacy and empowerment; rights according to different types of status as migrant; unmet expectancies; and conflicts of interest [13].

\subsection{Rationale for developing RLOs using the 'ASPIRE' framework}

On the basis of the core topics emerged from the needs analysis, an aggregation and prioritization process was then followed by each partner of the consortium in order to repurpose the learning content taking into account different aspects, including specific area of significance, knowledge expertise and access in network of related stakeholders. The participatory methodology of the "ASPIRE" framework was deployed in this process, and given the timeline of the project, focus in this study is given on the two first phases. According to this framework, the initial stage is to identify the objectives of the RLO and reflect on end-users' needs, in order to ensure that the appropriate objectives have been designed [8]. To this end, repurposing in Greece was focused on the development of an RLO on the support and empowerment of mental health and depression management of women refugees, entitled Maternal depression: Break the silence. In the second phase of the ASPIRE framework, which is the storyboarding workshop, stakeholders are invited to co-design and co-create the RLO content, a significantly important process, where justification of the needs and expectations among the main stakeholders is enabled [14]. Thus, an interactive storyboarding workshop was conducted, where experts and healthcare professionals were invited to provide their feedback and generate a recommended core content for our RLO.

\section{Results}

A storyboarding co-creation workshop, based on the inclusive participatory approach of the "ASPIRE"' framework, was virtually conducted by the Medical Physics Laboratory from the Aristotle University of Thessaloniki (AUTH) in December 2020, in order to generate the content of the RLO. In particular, 9 stakeholders participated in the workshop, with ages ranging from 21 to 56 years old, 2 research associates of the MedPhys Lab, 1 learning technologist, 2 partners of the project, 3 people working in the support of refugees and 1 refugee. This process was to ensure that stakeholders would actively contribute to the storyboarding workshop and provide input based on their experiences. At the end of the storyboarding workshop, qualitative and quantitative feedback was gathered from the stakeholders through an online questionnaire administered in Google Forms. The questionnaire comprised several statements regarding their experience in the workshop, and, in particular, 5-point Likert scale responses and open-ended questions were used. Overall, the majority of comments related to the workshop were exceptionally positive. According to stakeholders' feedback, the workshop strongly met their expectations and considered that its objectives were highly achieved. Additionally, they reported that they gained greater insight into maternal depression and they became familiarised with the storyboarding, the RLO development process and new online tools, for instance: 
"Gained more information on how the RLO should be formed, what to include, what is important.",

"I learned a lot about the subject of the case we were considering. It was my first try for the online storyboard tool.",

Participants found the workshop engaging, they expressed their ideas freely while the majority commented on how much they enjoyed the storyboarding workshop. They also reported that collaborating with a multidimensional team allowed for the greater coverage of the topic and the content development process, and, rated positively particular aspects of the process:

"Multidimensional collaboration-participated people from different domains.", "The distance brainstorming from different professionals/advisors." "Interactivity - sharing experiences " \& 'Interactions and collaboration."

Stakeholders provided feedback on how the workshop could be improved in future. Some of them commented that more familiarization with the storyboarding process is required, that could be achieved through a second session or a brief introductory meeting, while others highlighted that more refugees could be involved. The latter needs to be weighed carefully, due to the fact that involving more people may result in the possibility of having difficulty in achieving a consensus during discussion or in the event of conflicting opinions. At the end of the workshop, all participants received a "'certificate of attendance'".

\section{Discussion}

E-learning has been highlighted as a significant way of offering flexible learning to refugees, however, little research has been done assessing its impact, while there is a need to bridge the knowledge gap of digital health applications for refugees [3]. In this study the RLO development process for refugees' health integration is discussed, while the important role of the involvement of multiple stakeholders in a participatory process of conceptualizing digital learning and its subsequent design is designated. This methodology is based on the "ASPIRE" framework by involving all relevant stakeholders as co-creators in the storyboarding session. Essential feedback and enlightening ideas were expressed resulting in the successful apprehension of refugees' educational needs in regard to the healthcare system.

\section{Conclusions}

RLOs represent e-learning resources with a great potential for use within the refugees' healthcare integration setting. The "ASPIRE" framework adopted during the RLO development, offers the potential to engage a range of stakeholders in the creation of meaningful learning content and supplies a powerful, user-centered framework for RLO development. Hence, in order to ensure sustainability and increase potential impact of RLOs, it is crucial to include appropriate pedagogical design and adhesion to the learning objectives. 


\section{Acknowledgements}

This research has been partially funded by ERASMUS+ under the ReHIn project (reference number: 2019-1-SE01-KA204-060563). The authors would like to thank all the partner universities: Karolinska Institutet, University Of Nottingham and University of Valencia for the collaboration and also all stakeholders for their participation and contribution in this research.

\section{References}

[1] Shortall CK, Glazik R, Sornum A, Pritchard C. On the ferries: the unmet health care needs of transiting refugees in Greece. International health. 2017 Sep 1;9(5):272-80.

[2] UNHCR. Global trends: Forced Displacement in 2017. Geneva: United Nations High Commissioner for Refugees, 2018.

[3] Liem A, Natari RB, Jimmy, Hall BJ. Digital health applications in mental health care for immigrants and refugees: A rapid review. Telemedicine and e-Health. 2020 Jun 4;27(1):3-16.

[4] WHO. Re-profiling emergency medical services in Greece; 2017, Available at http://www.euro.who.int/en/health-topics/Health-systems/health-services-delivery/publications/2017/reprofiling-emergency-medical-services-in-greece-2017, Accessed Jan 15, 2021.

[5] WHO. Digital health: Seventy-first World Health assembly. 2018, Accessed at https://apps.who.int/gb/ebwha/pdf_files/WHA71/A71_R7-en.pdf, Accessed Jan 15, 2021.

[6] UNHCR. 2015 likely to break records for forced displacement - study. Geneva: United Nations High Commissioner for Refugees, 2015, Available at http://www.unhcr.org/5672c2576.html, Accessed Jan 15, 2021.

[7] Kumar N, Anderson RJ. Mobile Phones for Maternal Health in Rural India. Proceedings of the 33rd Annual ACM Conference on Human Factors in Computing Systems;2015 Apr 18-23;Seoul, Korea. p. $427-436$.

[8] Hassan N, Sellappans R, Chen PY, Yap WH, Ayub E, Konstantinidis ST, Poussa C, Wharrad HJ, Taylor MG. Participatory Approach in Reusable Learning Object (RLO) Development Using ASPIRE Framework: Taylor's University's Experience. In: Nair P, Keppell MJ, Lim CL, Mari T, Hassan T. Transforming Curriculum Through Teacher-Learner Partnerships, IGI Global;2020 p. 90-104.

[9] ReHIn project. Refugees Health Integration project (ReHIn), Available at https://ki.se/en/lime/rehin-aresearch-project-on-refugees-health-integration, Accessed March 2, 2021.

[10] Ruiz JG, Mintzer MJ, Issenberg SB. Learning objects in medical education. Medical teacher. 2006 Jan 1;28(7):599-605.

[11] Çinici MA, Altun A. Reusable content matters: a learning object authoring tool for smart learning environments. Smart Learning Environments. 2018 Dec 1;5(1):10.

[12] Wharrad H, Windle R, Taylor M. Designing digital education and training for health. In Digital Innovations in Healthcare Education and Training 2020 Sep 8. Academic Press. p.31-45.

[13] Stathakarou N, Konstantinidis S, Wharrad H, Spachos D, Bamidis PD, Tortajada LA, Salcedo VT and Karlgren K. Co-creating digital learning resources to enhance refugees' health integration in Europe. Proceeding of the 14th International Technology, Education and Development Conference (INTED2020); 2020 Mar 2-4; Valencia, Spain, IATED. p. 3799-380.Lubicz-Nawrocka T. From partnership to self-authorship: the benefits of co-creation of the curriculum. International Journal for Students as Partners. 2018 May 1;2(1):47-63.

[14] Talhouk R, Mesmar S, Thieme A, Balaam M, Olivier P, Akik C, Ghattas H. Syrian refugees and digital health in Lebanon: Opportunities for improving antenatal health. Proceedings of the $2016 \mathrm{CHI}$ Conference on Human Factors in Computing Systems; 2016 May 7-12;San Jose, CA, USA. p. 331-342. 